\title{
Occipital Neuralgia in Chiari I Malformation: Two Different Events or Two Different Faces of the Same Event?
}

\author{
Giacomo Tondo $^{1}$, Fabiola De Marchi ${ }^{1}$, Daniela Mittino ${ }^{1}$, Roberto Cantello $^{1}$ \\ 1. Department of Translational Medicine, Neurology Unit, Azienda Ospedaliero Universitaria "Maggiore \\ della Caritá", Novara
}

$\square$ Corresponding author: Giacomo Tondo, giacomo.tondo85@gmail.com Disclosures can be found in Additional Information at the end of the article

\section{Abstract}

Occipital neuralgia $(\mathrm{ON})$ is characterized by severe pain in the occipital region due to an irritation of the occipital nerves. Traumatic injuries, mass or vascular compression, and infective and inflammatory processes could cause ON. The dislocation of a nerve/muscle/tendon, as can happen in malformations such as the Chiari I malformation (CIM), also can be responsible. Usually, headaches associated with CIM and ON are distinguishable based on specific features of pain. However, the diagnosis is not easy in some cases, especially if a clear medical history cannot be accurately collected. Determining if the pain is related to ON rather than to CIM is important because the treatments may be different.

Categories: Neurology, Pain Management, Neurosurgery

Keywords: occipital neuralgia, arnold chiari malformation

\section{Introduction}

Received 08/18/2017

Review began 08/23/2017

Review ended 11/24/2017

Published 11/29/2017

\section{(c) Copyright 2017}

Tondo et al. This is an open access article distributed under the terms of the Creative Commons Attribution License CC-BY 3.0., which permits unrestricted use, distribution, and reproduction in any medium, provided the original author and source are credited.
Occipital neuralgia (ON) is a headache characterized by severe, paroxysmal, stabbing, or shooting pain in the area of the greater, lesser, or third occipital nerve. Pain, unilateral or bilateral, originates in the occipital region; it may be associated with dysesthesia or allodynia due to pressure stimulation of the scalp. The distribution is due to the emergence of the greater occipital nerve over the $\mathrm{C} 2$ dermatomes [1]. It is described as a rare form of headache; nevertheless, in a recent paper, authors consider it as the third-most common headache syndrome, following migraines and tension-type headaches [2]. Other symptoms may be associated with the headache such as visual disturbances, nausea, dizziness, and tinnitus. Etiology includes trauma, compression due to vascular abnormalities and mass lesions, inflammatory and infective processes, fibrositis, myositis, or the dislocation of nerve/muscle/tendon structures due to arthrosis, sclerosis, osteolytic lesions, and Chiari I malformation (CIM) [3]. Treatment includes antidepressants, antiepileptics, infiltration of the nerve (anesthetic and corticosteroids), and botulinum toxin A. Diagnosis is clinical and is inferred from the response to local anesthetic block. We encountered a particularly complex case of ON, mainly because of difficulties in obtaining reliable clinical data.

\section{Case Presentation}

A 44-year-old Togolese female presented to our headache center for refractory pain, which began three years ago. Her medical history was difficult to gather due to language problems. 


\section{Cureus}

The patient complained of persistent occipital and cervical pain, with occasional paroxysmal attacks of stabbing pain accompanied by nausea and dizziness. No psychophysical comorbidity, no cigarette or alcohol use, or hormone therapies were described. A brain computed tomography (CT) scan with an angiographic study was normal. A neurologic examination revealed no abnormalities. A physical examination demonstrated paraspinal neck extensor tension. Amitriptyline (30 mg/day) was started, without benefit. An inconsistent response was obtained with nonsteroidal anti-inflammatory drugs. Six months later, a new therapeutic approach with flunarizine was attempted, but it was ineffective as well. Later, a prophylaxis with topiramate was initiated but was interrupted because of mood changes. At the same time, a brain magnetic resonance imaging (MRI) revealed a lowering of the cerebellar tonsils, compatible with CIM (Figure 1). However, the pain features were not compatible with CIM headaches because there was no postural change and the Valsalva or similar maneuvers did not exacerbate the symptoms. On neurological examination, we noticed a Tinel sign, on percussion of a region about $3 \mathrm{~cm}$ near the occipital protuberance, evocative of $\mathrm{ON}$. Thus, the patient was referred to the pain clinic where she underwent treatment with two anesthetic blocks of the greater occipital nerve. This led to rapid and complete regression of the painful symptomatology.

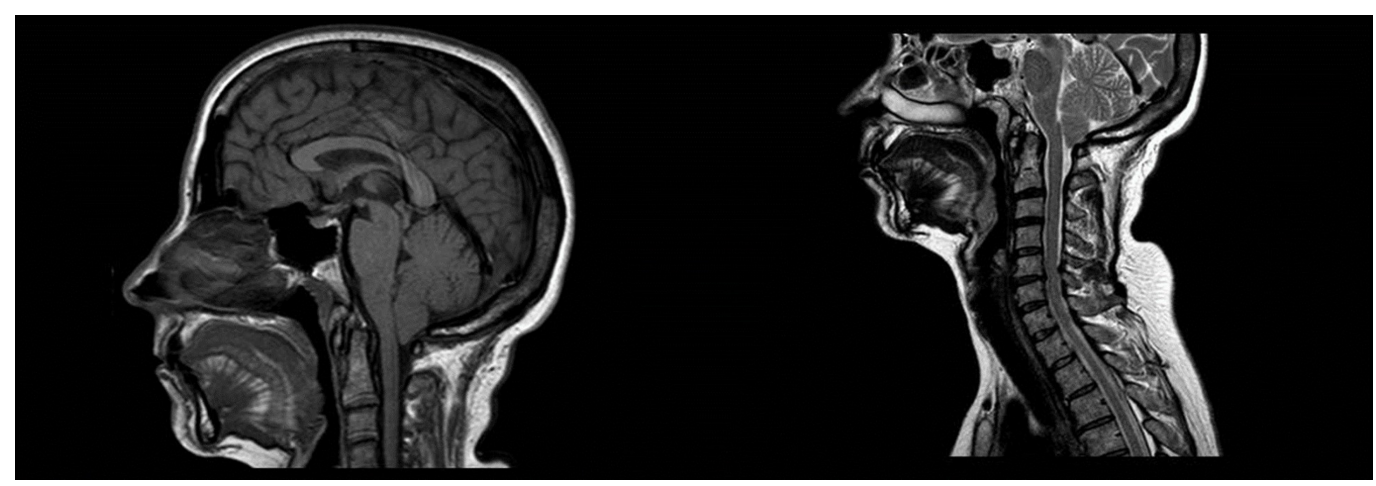

\section{FIGURE 1: Brain MRI showing Chiari I malformation}

Brain MRI T1 (left) and T2 (right) weighted sagittal sequences show a Chiari I malformation. The tonsillar descent is about $8-8.5 \mathrm{~mm}$.

MRI: magnetic resonance imaging

\section{Discussion}

ON is a debilitating headache localized in the occipital area. It can occur in cases of irritation, compression, or traction of the occipital nerves, as in the pathologic processes of the neck vessels, bones, muscles, or tendons. Association with CIM is rarely reported [4], but the prevalence of a simultaneous occurrence of the two diseases is unknown. In addition, the pathogenesis of headaches with CIM is unclear. CIM is reported to be related to dislocation/irritation of other cranial nerves when surgery can be healing [5-6]; however, we do not have a series report of the benefit of surgery in cases of ON in CIM. Moreover, it must be considered that both conditions could cause headaches in the same region, even with specific characteristics that are not always easily identified; this can make diagnosis a challenge.

In case of a suspicion of both CIM and ON, it is useful to consider the diseases as two different events. Indeed, until now, no conclusive data on a causative link between ON and CIM have been reported in the literature. For this reason, it is reasonable to try to treat ON with local 
anesthetic blocks before the surgical decompression of CIM, especially if the pain is stabbing or shooting, there is hypo/anesthesia or dysesthesia in the occipital region, and pain is not exacerbated by a cough, postural variation, or the Valsalva maneuver.

\section{Conclusions}

In the present case, the language barrier and the finding of CIM on an MRI scan had originally confounded the origin of pain. This case emphasizes the importance of a conservative treatment approach in patients with clinical features of ON and an occasional finding of CIM. In these cases, treatment of ON with localized blocks may help patients with headaches, who will respond without surgery even in the presence of radiologic findings of CIM.

\section{Additional Information \\ Disclosures}

Human subjects: Consent was obtained by all participants in this study. Conflicts of interest: In compliance with the ICMJE uniform disclosure form, all authors declare the following: Payment/services info: All authors have declared that no financial support was received from any organization for the submitted work. Financial relationships: All authors have declared that they have no financial relationships at present or within the previous three years with any organizations that might have an interest in the submitted work. Other relationships: All authors have declared that there are no other relationships or activities that could appear to have influenced the submitted work.

\section{References}

1. Headache Classification Subcommittee of the International Headache Society: The international classification of headache disorders: 2nd edition. Cephalalgia. 2004, 24:9-160.

2. Liu A, Jiao Y, Ji H, Zhang Z: Unilateral occipital nerve stimulation for bilateral occipital neuralgia: a case report and literature review. J Pain Res. 2017, 20:229-232. 10.2147/JPR.S125271

3. Vanelderen P, Lataster A, Levy R, Mekhail N, van Kleef M, Van Zundert J: Occipital neuralgia. Pain Pract. 2010, 10:137-144. 10.1111/j.1533-2500.2009.00355.x

4. Cesmebasi A, Muhleman MA, Hulsberg P, Gielecki J, Matusz P, Tubbs RS, Loukas M: Occipital neuralgia: anatomic considerations. Clin Anat. 2015, 28:101-108. 10.1002/ca.22468

5. Colpan ME, Sekerci Z: Chiari type I malformation presenting as hemifacial spasm: case report. Neurosurgery. 2005, 57:E371. 10.1227/01.NEU.0000166688.69081.8B

6. Yglesias A, Narbona J, Vanaclocha V, Artieda J: Chiari type I malformation, glossopharyngeal neuralgia and central sleep apnoea in a child. Dev Med Child Neurol. 1996, 38:1126-1130. 10.1227/01.NEU.0000166688.69081.8B 